\title{
Defect Detection in Thick Aircraft Samples Based on HTS SQUID-Magnetometry and Pattern Recognition
}

\author{
K. Allweins, G. Gierelt, H.-J. Krause, and M. v. Kreutzbruck
}

\begin{abstract}
SQUID technology has recently evolved to the point that it can be used for industrial applications in Non-Destructive Evaluation (NDE). We present the implementation of an HTS SQUID magnetometer in an eddy current testing system to measure very thick structures in large aircraft. We measured a 62 mm-thick, bolted aluminum sample from EADS-Airbus, similar to the three-layered outer wing splice that is being proposed for the Airbus A-380. The combination of field sensitivities of a few $\mathrm{pT} / \sqrt{ } \mathrm{Hz}$ and a large dynamic range of about $140 \mathrm{~dB} / \sqrt{ } \mathrm{Hz}$ enabled us to detect defects at a depth of up to $40 \mathrm{~mm}$. However a problem was presented by the fact that deep-lying defects which caused small field variations were superimposed on field changes, in their turn caused by current distortions in the vicinity of the titanium bolts. Separation of these two contributions was achieved through parameter optimization based on FEM simulations and signal processing. We report on the possibilities for flaw detection using adapted eddy current excitation.
\end{abstract}

Index Terms-Eddy current testing, HTS SQUID.

\section{INTRODUCTION}

$\mathbf{O}$ VER the past few years, sensitive magnetic field sensors have been implemented with remarkable success in eddy current testing (ECT). Conventional techniques using inductive coil systems to measure the magnetic field response have been gradually replaced by alternative sensors, such as flux-gates [1], giant magnetoresistive sensors and SQUIDs [2], [3]. HTS SQUIDs provide a combination of high field sensitivity at low frequencies and an outstanding high dynamic range. This is especially important for the detection of minute or deep lying defects in the structure, component or material being tested [4].

Although magnetic sensors have a low intrinsic noise level, they do not automatically guarantee reliable crack detection. Because of their high degree of sensitivity, they measure all electromagnetic interference. In the case of an EC measurement, interference can arise from three sources: i) external noise, though this is the least significant factor, ii) EC effects created by the sensor head's artificial movement (e.g. vibration, tilting, and Lift-Off effect [5]), and iii) EC artifacts arising from the sample itself.

The latter accounts for the most significant noise factor in the case of complex samples. Due to the skin effect, difficulties arise when testing samples which include edges, holes or a number of different materials close to their surface. The induced

Manuscript received August 6, 2002. The work was supported by the German Ministry of Education and Research under Grant 13N7427/9.

K. Allweins, G. Gierelt, and M. v. Kreutzbruck are with the Institute of Applied Physics, Justus-Liebig-University Giessen, 35392 Giessen, Germany.

H.-J. Krause is with the Institute of Thin Films and Interfaces (ISG),

Forschungszentrum Jülich, D 52425 Jülich, Germany.

Digital Object Identifier 10.1109/TASC.2003.813696

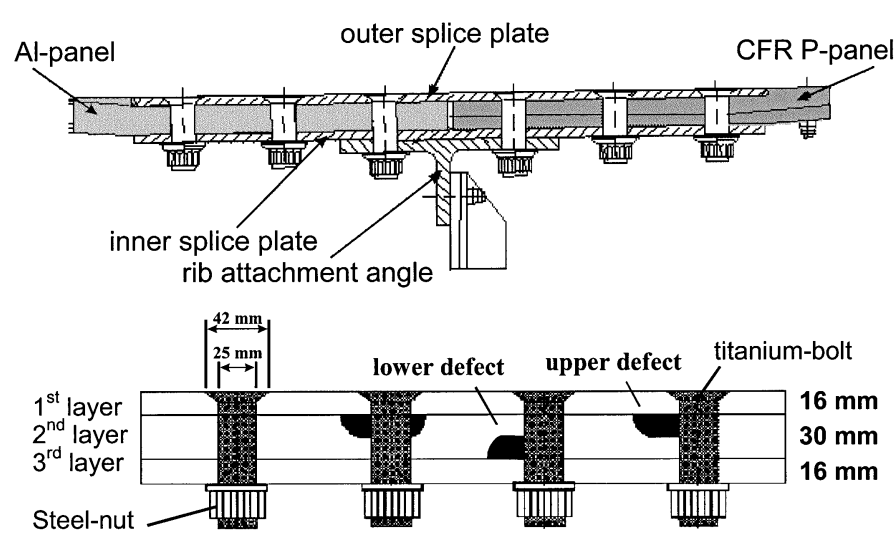

Fig. 1. Sketch of a projected A-380 wing splice.

EC density is higher at the sample's surface than it is at deeper levels, giving rise to significant distortion currents at the surface near components with differing electrical conductivity. This in turn results in strong variations of the magnetic field distribution above the sample's surface. Thus in many NDE applications the SQUID's high field sensitivity can only be exploited through the use of additional signal processing, whereby defect-induced field variations are separated from any type of superimposed background signals.

To prevent EC interference caused by the make-up of the sample itself, the detection of deep-lying cracks requires the additional use of sophisticated excitation schemes with adapted coil geometries to decrease the EC density at the sample's surface. A new approach is described in Part 4, in which an excitation coil system is used to reduce surface currents and enhance EC density in deeper material levels by adapting superimposed excitation fields. This reduces the influence of bolts or rivets inside the sample and allows for easier defect signal extraction from the superimposed back ground.

\section{Measurement Configuration And TeSt SAmple}

For the examination of extremely thick aircraft parts we were provided by EADS-Airbus with several multi-layer samples resembling a part of the wing splice planned for the new Airbus A-380. The wing splice connects the outer wing (CFR-P-compound) with the inner wing (aluminum alloy) and consists of three layers of aluminum with a total thickness of $62 \mathrm{~mm}(16$, $30,16 \mathrm{~mm}$ ). The layers are bolted together with $25 \mathrm{~mm}$ dia. titanium bolts, the upper parts of which are conical shaped and have a diameter of $42 \mathrm{~mm}$ at the sample's surface (Fig. 1). The second layer contains an exchangeable sheet, into which defects of differing lengths $\ell$ were introduced adjacent to the bolt at 


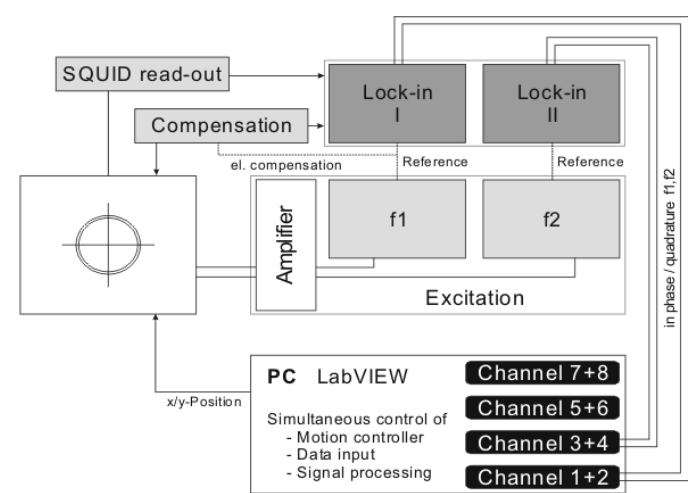

Fig. 2. Sketch of the measurement configuration.

two different depths; one at 16-31 mm ("upper" defect) and another at 31-46 mm ("lower" defect). The conductivity of the aluminum was about $20 \mathrm{MS} / \mathrm{m}$, whereas the conductivity of the bolt, made from titanium alloy, was more than one order of magnitude lower.

In our measurements we used two excitation frequencies. One was used to provide depth selective information about the defect. The second was used to localize the bolt's position to make it easier to differentiate between bolt and defect signals.

Fig. 2 shows the principle block diagram of the measurement configuration. The excitation unit generates signals of frequency $f_{1}(<100 \mathrm{~Hz})$ and $f_{2}(>3 \mathrm{kHz})$, each of which is fed to an individual inductor to excite eddy currents in the sample. We used two single wires as excitation coils for frequency $f_{1}$ and $f_{2}$. The wires were perpendicular to each other, their crossover located below the center of the cryostat.

The SQUID was located right above the crossover of the two wires, which were attached to a small $x / y$-stage in between the cryostat and the sample, and positioned so that the excitation field at the SQUID was as low as possible.

Two lock-in amplifiers measured the amplitude and phase of the SQUID readout output at the two excitation frequencies.

In our measurements, we used HTS-rf-washer SQUID magnetometers [6] biased at $630 \mathrm{MHz}$. The field sensitivity of the washer SQUID was about $1 \mathrm{pT} / \sqrt{ } \mathrm{Hz}$ at signal frequencies above $10 \mathrm{~Hz}$, and the field to flux transfer coefficient was about $15 \mathrm{nT} / \Phi_{0}$. The SQUID was cooled using a Joule-Thomson cooler. The spacing between SQUID and sample was determined to be $5.5 \mathrm{~mm}$ while the size of the SQUID washer was 2.5 $\mathrm{mm}$. Further information on the cooling environment, SQUID integration and experimental setup can be found elsewhere [3].

\section{MEASUREMENTS}

Fig. 3 shows the measured field distribution along a line scan across the bolt, when using wire excitation. The wire was aligned perpendicular to the $x$-axis and the optimum excitation frequency was determined to be $38 \mathrm{~Hz}$ (by FEM simulations). The defect signal was much smaller than the superimposed bolt signal and thus cannot be seen in the raw data (Fig. 3, line 1).

In order to separate the defect signal from the background, two steps in signal processing had to be taken. Fig. 3 shows the field resulting from these signal processing steps. First, the Lift-Off effect [5] (change of response field due to changes in

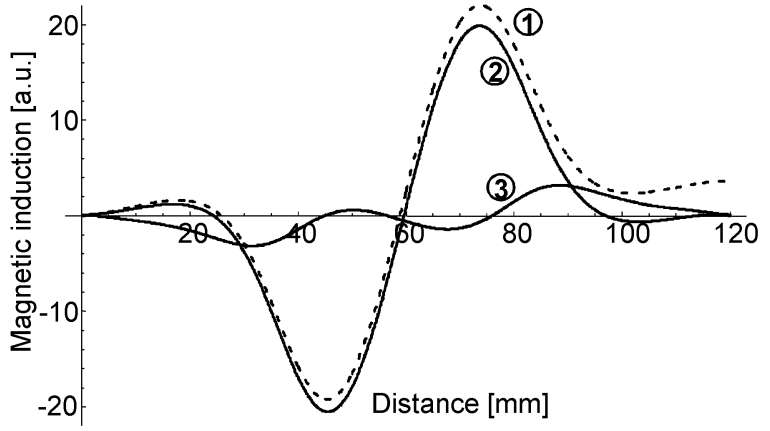

Fig. 3. Line scan, across a bolted multi-layered sample using $38 \mathrm{~Hz}$ excita tion frequency. Line 1: raw data containing both bolt and defect signal. Line 2: Data after suppression of the lift-off effect by subtracting a linear function. Line 3 : Data after suppression of the bolt signal by rotating the phase.

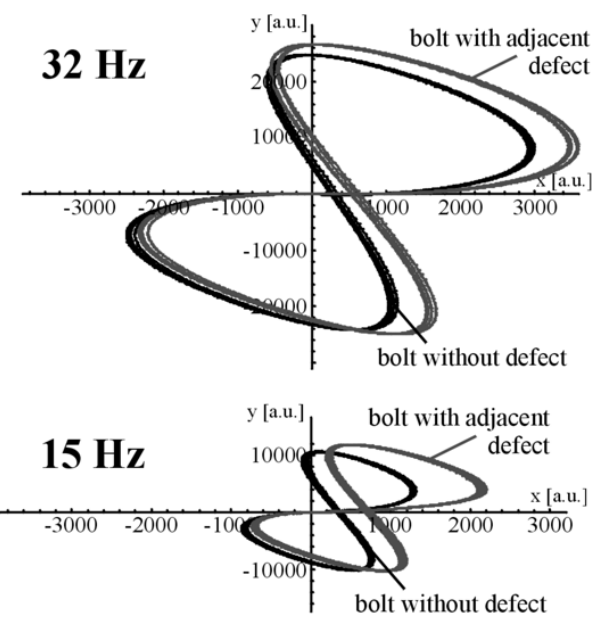

Fig. 4. Ten trajectories of line scans across a bolt without defect and with a "lower" defect, $y$-axis: quadrature channel; $x$-axis: in-phase channel. Frequency: $32 \mathrm{~Hz}, 15 \mathrm{~Hz}$; defect length: $30 \mathrm{~mm}$ HTS SQUID, using a bandwidth of $30 \mathrm{~Hz}$.

the spacing between SQUID and sample) had to be eliminated by subtracting a linear function (see Fig. 3, line 2). The second step contained a phase rotation to minimize the bolt signal and enhance the defect signal (see Fig. 3, line 3).

In conventional EC-signal processing, both the in-phase channel and the quadrature channel are usually combined into a complex plane-like diagram, which makes the interpretation of eddy current trajectories easier. Due to the undertaken adapted phase rotation, the defect signal mainly occurred in changes along the $x$-axis. This is shown in Fig. 4, where ten trajectories of bolts without adjacent defects and bolts with $30 \mathrm{~mm}$ long "lower" defects are represented in a complex plane diagram.

In practice the reduction of the bolt signal in the in-phase channel of the lock-in amplifier is limited to a factor of between 5 and 30, depending on the excitation frequency and the shape of the excitation coil. In the case of lower defects, the bolt accounts for the major part of the in-phase response ( $x$-axis), despite the adapted phase rotation. This super imposed bolt signal is caused by field distortions emanating from the part of the bolt that lies at the same depth as the defect. In this way the bolt signal has the same phase shift as the defect signal. Even so, we were still able to detect defects with a length of about $10 \mathrm{~mm}(20 \mathrm{~mm})$ located at a depth of 16-31 mm (31-46 mm). 


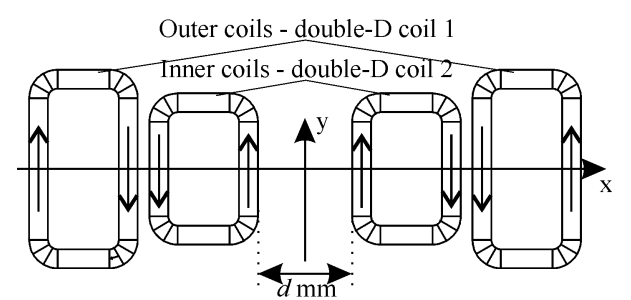

Fig. 5. Dual-gradiometric coil system (DGC) consisting of 2 double-D-coils. The direction of the excitation current is indicated by the arrows along the coil windling.

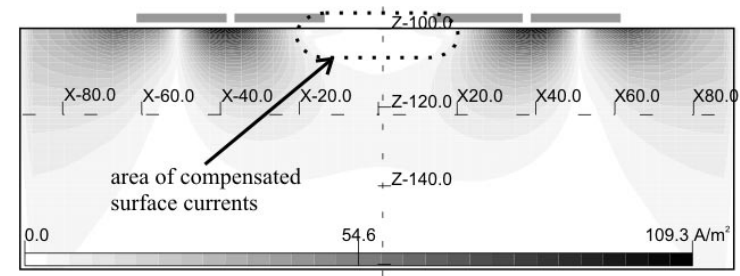

Fig. 6. Induced eddy current density in the sample at an excitation frequency of $10 \mathrm{~Hz}$ and a distance $d$ of $28 \mathrm{~mm}$, calculated by FEM-simulations.

The measurements were carried out with different excitation frequencies. As expected from the induction law, the eddy current response is reduced when using lower frequencies. It turned out that in practice the optimum excitation frequency was somewhat lower than the calculated values when using wire excitation instead of excitation with a circular coil.

The maximum defect signal for the "lower" defect was obtained by using frequencies of about $20 \mathrm{~Hz}$. But sometimes it is sensible to use smaller frequencies, which give rise to a larger ratio of defect signal and bolt signal, to enable an easier extraction of the defect signal (Fig. 4, $15 \mathrm{~Hz}$ ). However, other eddy current effects have to be taken into consideration, e.g., the edge effect, which could inadmissible interfere with the EC measurements if frequencies are too low.

\section{ADAPTED EDDY CURRENT EXCITATION}

To minimize EC interference caused by the sample's own components, i.e., a reduction of induced surface currents, we adapted a Dual Gradiometric Coil excitation scheme (DGC), consisting of two double-D coils (see Fig. 5). Both double-D coils were applied with the same frequency but driven with two different excitation currents $\left(J_{1}, J_{2}\right)$ and phase shifts $\left(\varphi_{1}, \varphi_{2}\right)$. By using 3-D Finite Element simulations we determined values for $J_{1} / J_{2}$ and the phase difference $\Delta \varphi=\varphi_{1}-\varphi_{2}$ that compensated the induced eddy current density at the sample's surface $(x=-20 \mathrm{~mm}$ to $+20 \mathrm{~mm}, z=0 \mathrm{~mm})$. The outer coil's current $J_{2}$ was 3,7 times higher than $J_{1}$ when using a distance $d$ of $28 \mathrm{~mm}$ and an excitation frequency of $10 \mathrm{~Hz}$. Due to an adapted phase setting $\Delta \varphi$ the surface currents were even significantly reduced below the inner part of the inner double-D coil (see Fig. 6).

Fig. 7 shows the induced EC density at different depths along the $z$-axis in the sample. The greater the depth, the larger the EC density, which reaches the maximum at a specific depth (here at $22 \mathrm{~mm}$ ). The depth at which maximum induced currents occur depends on the applied frequency and the distance $d$. In comparison, the double-D coil's graph is plotted showing a maximum

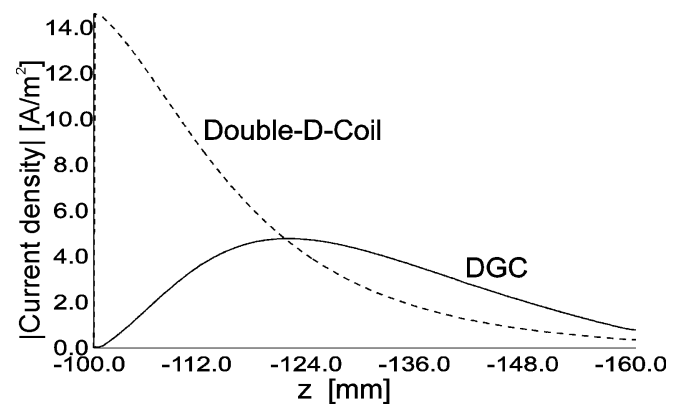

Fig. 7. Induced EC density on the $z$-axis of the sample $(x=0 \mathrm{~mm}, y=$ $0 \mathrm{~mm})$.

(a)
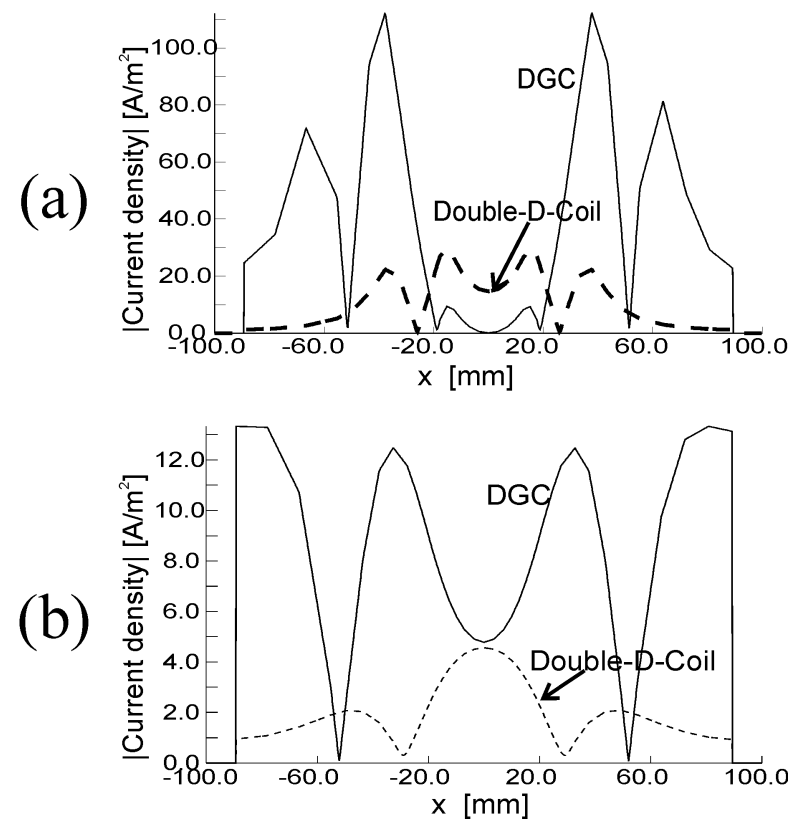

Fig. 8. Induced eddy current density on the $x$-axis on the surface of the sample (a) and at a depth of $z=21 \mathrm{~mm}$ (b).

eddy current density at the surface of the sample and a continual attenuation for deeper levels.

The induced EC density reaches its peak along the $x$-axis, after a certain distance from the center where EC density has practically vanished [Fig. 8(a)]. The curve shape is similar at a depth of $z=21 \mathrm{~mm}$, but through the use of our DGC the EC density at regions close to the center only dropped to half the values of the peaks to the left and right [Fig. 8(b)]. Because of these peaks, a defect located adjacent to the bolt gives rise to large distortion currents and is thus easier to detect. At the same time, the minimum EC density at the center reduces the influence of the rivet or bolt itself.

To confirm the 3-D FEM simulations, we measured the magnetic field variation $\left|B_{z}\right|$ above an aluminum sample containing a $20 \mathrm{~mm}$ long surface defect. The SQUID-magnetometer was placed right above the center of the coil system, where the excitation field is minimal. With the optimal setting for $J_{1} / J_{2}$ and $\Delta \varphi$, the magnetic field variation above the sample was minimized in the area ranging from $-20 \mathrm{~mm}$ to $20 \mathrm{~mm}$. An uncompensated setting is obtained by subtracting $180^{\circ}$ from the proper value of $\Delta \varphi$, which yields a maximal response of the surface defect (Fig. 9). The results show that it is possible to compensate signature of structures located near the surface. 


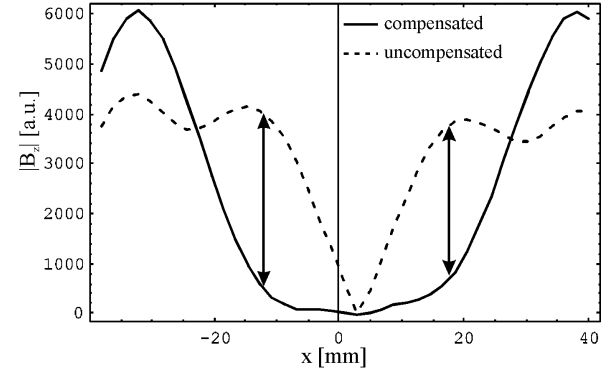

Fig. 9. Measurement of a $20 \mathrm{~mm}$-long surface defect for an optimal setting of $J_{1} / J_{2}$ and $\Delta \varphi$ and an uncompensated setting of $\Delta \varphi-180^{\circ}$ (excitation frequency: $38 \mathrm{~Hz}$, distance $d: 28 \mathrm{~mm}$ ). The arrows indicate the area where the induced eddy current density on the surface of the sample is reduced.
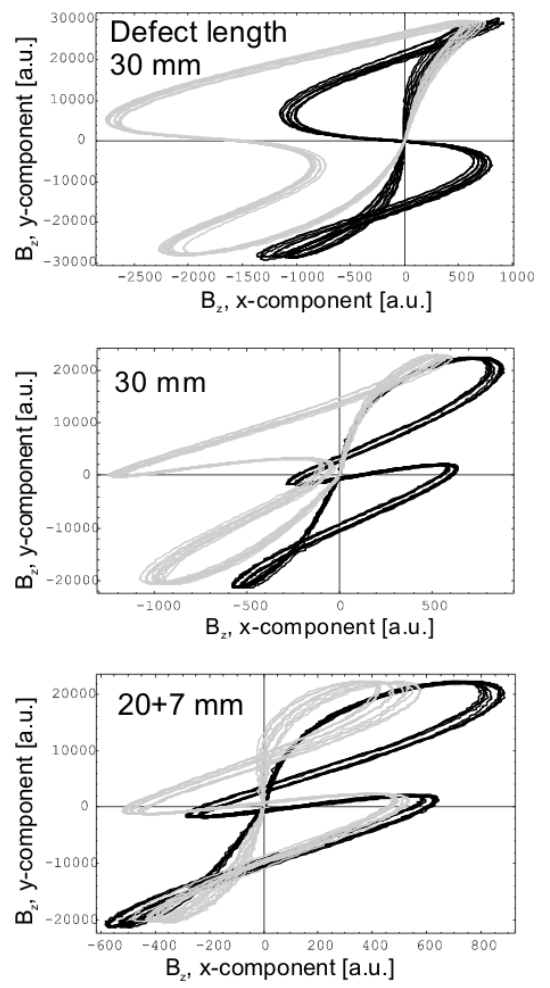

Fig. 10. Measurements of bolts with adjacent cracks of different lengths at different depths (gray: bolt with adjacent crack, black: defectless bolt), 10 measurements for each bolt. (a) $38 \mathrm{~Hz}$, defect depth 16-31 mm; (b) $15 \mathrm{~Hz}$, defect depth 16-31 mm; (c) $15 \mathrm{~Hz}$, defect depth 31-46 mm.

By using the optimal settings for $J_{1} / J_{2}$ and $\Delta \varphi$ we carried out measurements on the A380 wing splice samples. The "upper" defect at a depth of 16-31 mm was measured with an adapted excitation frequency of $38 \mathrm{~Hz}$, the "lower" defects at a depth of 31-46 mm were detected with a frequency of $15 \mathrm{~Hz}$.

Because of adapted phase rotation inside the complex plane-like diagram, the defect signal mainly occurred in changes along the $x$-axis. Compared to measurements with a single double-D coil, the shape of the trajectories was more complicated. However, a higher deviation from the reference bolt signal could be recognized when using an excitation with the DGC.

The "upper" defect can be detected easily with both excitation frequencies [Fig. 10(a) and (b)]. The deepest-lying defect at the depth of 31-46 mm caused only a relatively small field variation [Fig. 10(c)], due to too small a distance $d$ inside the inner double-D coil. The maximum value of the induced EC arises at a depth of about $20 \mathrm{~mm}$, while the defect is located at a depth of 31-46 mm. However, the defect signal can be easily separated from the reference bolt signal through, for example, the extraction of different signal features. Compared to other excitation methods, the DGC offers two key advantages: a higher amplitude in the in-phase channel and signal shapes that are easier to differentiate from the bolt signal.

\section{CONCLUSION AND OUTLOOK}

The use of SQUID technology involves high costs and complex handling compared to alternative field sensors. Nevertheless, SQUIDs have been successfully employed in several NDE application such as magnetics and eddy current testing when conventional methods failed to provide sufficient reliability.

Successful defect detection in thick aircraft parts, such as the planned wing splice of the Airbus A-380, was shown as an example in which SQUID sensors can be applied in current NDE applications. Another area in which the implementation of such technology holds promise is laser weld testing, which requires high field sensitivity to detect small shrink holes hidden within deeper regions. Similar to riveted aircraft samples, welds have an uneven surface which inevitably decreases the reliability of EC crack detection. Here, the combination of a sensor's high field sensitivity and sophisticated excitation geometry, such as shown in Part 4, could be key in detecting small and deeplying material defects. Coupling SQUIDs with adapted excitation schemes enables one a "deeper" look into samples and offers the potential to solve a range of NDE problems.

\section{ACKNOWLEDGMENT}

The authors thank W.-B. Klemmt (EADS Airbus) and W. Becker (Lufthansa Technik) for their contributions.

\section{REFERENCES}

[1] J. Pavo et al., "Eddy current testing with fluxset probe," in Studies in Applied Electromagnetics and Mechanics. Amsterdam, The Netherlands: IOS Press, 1997, vol. 12, pp. 215-222.

[2] J. P. Wikswo, Jr., "SQUID magnetometers for biomagnetism and nondestructive testing: Important questions and initial answers," IEEE Trans. Appl. Supercond., vol. 5, no. 2, pp. 74-120, 1995.

[3] R. Hohmann, M. Maus, D. Lombarski, M. Grüneklee, Y. Zhang, H.-J. Krause, H. Bousak, and A. I. Braginski, "Aircraft wheel testing with machine-cooled HTS SQUID gradiometer system," IEEE Trans. Appl. Supercond., vol. 9, no. 2, pp. 3801-3804, 1999.

[4] M. Mück, C. Welzel, A. Farr, and F. Schölz, "Nondestructive testing of niobium sheets for superconducting resonators," IEEE Trans. Appl. Supercond., 2002, submitted for publication.

[5] "Nondestructive testing handbook," in Electromagnetive Testing, 2nd ed. Columbus, OH: American Society for Nondestructive Testing, 1996, vol. 4.

[6] Y. Zhang, M. Mück, K. Hermann, J. Schubert, W. Zander, A. I. Braginski, and C. Heiden, "Sensitive rf- SQUIDs and magnetometer operating at 77 K," IEEE Trans. Appl. Supercond., vol. 3, p. 2465, 1993. 